\title{
Nuclear Medicine and Wall Street: An Evolving Relationship
}

\author{
Mike Sherman $^{1}$ and Rachel Levine ${ }^{2}$ \\ ${ }^{1}$ Chimerix, Inc., Durham, North Carolina; and ${ }^{2}$ Communications, Advanced Accelerator Applications, USA, Inc., Millburn, \\ New Jersey
}

\begin{abstract}
Until recently, it has been challenging to engage Wall Street and large pharmaceutical companies in radiopharmaceutical opportunities. The modest economic prospects of most diagnostic radiopharmaceuticals have not attracted keen interest from the broader business community, despite the rapid advancement of diagnostic imaging capabilities and their increasingly crucial role in the therapeutic process. Similarly, compelling science supporting select radiopharmaceutical therapies in oncology has been overshadowed by the unique challenges posed by this class of drugs and historical commercial failures that serve as sobering reminders of risk. Fortunately, a few notable successes in the targeted radioligand therapeutic space are changing this dynamic, fueling a new flow of investor capital into these technologies and inciting increased merger and acquisition activity that has yielded significant value creation for investors. If the nuclear medicine industry is able to continue to effectively manage historical challenges, then there is significant opportunity for a new and promising wave of radioligand therapies to significantly change the oncology treatment paradigm and elevate the profile of the entire nuclear medicine sector.
\end{abstract}

Key Words: nuclear medicine; Wall Street; radioligand therapies; Advanced Accelerator Applications; Endocyte

J Nucl Med 2019; 60:20S-24S

DOI: 10.2967/jnumed.118.220798

\section{$\mathbf{S}$} attempted to use radiation to treat cancer and other diseases (1). The discovery of radium as a source of radiation by Pierre and Mary Curie just a few years later further incited interest in the area. Unfortunately, a lack of knowledge regarding radiation biology resulted in limited therapeutic benefits compared with side effects for these approaches (2). By the 1950s, advances in radiation physics and technology led to the widespread use of external-beam radiotherapy as a key treatment modality for cancer, alongside surgery (3). Although external-beam radiation remains one of the most efficient tools for eliminating isolated, discrete disease, its application in the treatment of more advanced, diffuse disease is quite limited.

In parallel to the development of external-beam radiotherapy in the early 20th century, certain nuclear medicine pioneers, such as Saul Hertz, experimented with the targeted therapeutic application of radionuclides, such as ${ }^{131} \mathrm{I}$, in thyroid disease (4).

Received Mar. 20, 2019; revision accepted Apr. 19, 2019.

For correspondence or reprints contact: Rachel Levine, Advanced Accelerator Applications, USA, Inc., 57 East Willow St., Millburn, NJ 07041.

E-mail: rachel.levine@adacap.com

COPYRIGHT (C) 2019 by the Society of Nuclear Medicine and Molecular Imaging.
However, no major advances in this area were to occur until years later, with the invention of the concept of peptide receptor radionuclide therapy in the late 1980s (5). Instead, the nuclear medicine field was focused on the development of novel diagnostic radiopharmaceuticals supporting new imaging technologies, such as tomographic imaging (6). The rapid growth and expansion of these diagnostic modalities over the ensuing decade drove the industry while Wall Street became fascinated by other emerging therapeutic trends.

Despite the singular focus of the nuclear medicine industry on the growth of diagnostic radiopharmaceuticals for several years, developments in antibody conjugate technologies led to the launch of antibody-targeted radiotherapeutics in the early 2000s. ${ }^{90}$ Y-ibritumomab tiuxetan (Zevalin; Spectrum Pharmaceuticals, Inc.) and ${ }^{131}$ I-tositumomab (Bexxar; GlaxoSmithKline) were the first among this new class of therapeutic agents to be approved, pairing the potency of radiation with the precision of antibody targeting for the treatment of some forms of non-Hodgkin lymphoma $(7,8)$. Clinical results for both agents were compelling, and the promise of these therapies piqued the interest of the investment community $(9,10)$. Investors were watching closely. Unfortunately, what they observed were "good" drugs that ultimately failed to achieve their commercial potential. These therapies fell short of expectations for a variety of reasons, including oncology referral incentives, drug administration challenges, confusion over appropriate patient populations, cost and complexity of manufacturing, and supply challenges $(11,12)$. Anyone seeking funding for new projects in this space, even until very recently, would be reminded of these failures. It is clear that there were still problems to resolve before this therapeutic concept could be advanced to the next level.

\section{THE STARS ALIGN}

Fortunately, several circumstances aligned in the years that followed, facilitating further expansion of the use of targeted, systemically administered radiation to treat cancer.

\section{Improvement in Small-Molecule Drug Targeting}

Crystallization methods have accelerated the development of optimized targeting ligands with a high affinity for cell surface targets on cancer cells (13). The use of such targeted small molecules, in conjunction with a stable chelator construct, enables the use of highly potent isotopes. This approach not only yields more efficient tumor penetration than what was achieved with previously used isotopes but also limits toxicities that have been associated with the extended circulation of large molecules. In addition, ligand manipulation has allowed further maximization of target binding time and tumor retention before clearance from the circulation (14). Extensive cataloging of viable cell surface proteins also supports the thesis that dozens of additional radiotherapeutic targets exist beyond those 
already being investigated; these include surface molecules without downstream activity, which is not required for radioligand therapy.

\section{Development of Radionuclides with Favorable Properties}

${ }^{177} \mathrm{Lu}$, a $\beta$-emitter, has a 6.65 -d half-life, allowing the delivery of on-target DNA-damaging radiation for a few days once administered (15). This half-life also provides some flexibility in the supply chain process. In addition, the relatively short path length of ${ }^{177} \mathrm{Lu}$ $\beta$-emissions minimizes collateral toxicity while allowing cross-fire cell killing in heterogeneous disease. ${ }^{225} \mathrm{Ac}$, an $\alpha$-emitter, has more recently emerged as a promising therapeutic radionuclide. $\alpha$-particles have a more potent cell-killing capability and a shorter path length than $\beta$-particles; however, clinical utility and production capacity are still being evaluated.

\section{Advances in New Processes for Efficient Manufacturing and Distribution of Radioligand Therapies}

As the commercial viability of these therapies has become clearer, the number of companies investing in new production processes and supply chain infrastructure has expanded. However, barriers to the widespread supply of certain radionuclides and manufacturing complexities remain and will need to be addressed by industry so that this therapeutic class can reach its full potential in investors' eyes.

\section{Development Advantages}

From a development standpoint, radioligand therapies (and nuclear medicine in general) offer some compelling advantages relative to certain other therapeutic approaches in oncology. The following factors help reduce the risks of development, enhancing the likelihood of success and attractive returns for investors.

Molecular imaging with radioligand diagnostics enables real time, whole-body validation of cellular targets in a safe setting, reducing patient risk in phase 1 studies. Molecular imaging is used to ensure sufficient targeted uptake of radioligand therapies in diseased cells and is increasingly being perceived as a tool to support development decisions early in the process (16). Imaging results also provide early insight into potential patient selection criteria-which may increase the likelihood of a response to radioligand therapy_as well as information that may affect overall treatment decisions. Imaging results can eliminate years of costly development in a patient-friendly manner.

Dosimetry analysis from serial patient imaging supports quantification of the presence of a drug in tumor and nontumor tissues. This analysis not only accelerates understanding of the potential therapeutic opportunity for radioligand therapy but also assists in anticipating potential toxicities and providing valuable information regarding pharmacokinetic and pharmacodynamic effects (16). Nuclear medicine is unique in its ability to quantify where a drug goes in such a specific manner. This characteristic is an exceptional advantage over those of most other therapeutic approaches, which rely on secondary indicators of systemic exposure (such as plasma), to provide an "inference" of the presence of a drug at sites of disease. Traditional oncology agents do not provide such information.

The different profiles of $\alpha$ - and $\beta$-emitting radionuclides offer flexibility in drug design and the opportunity to further tailor radioligand therapy to tumor or disease characteristics. For example, the longer path length of $\beta$-emitters may be better suited for a disease with target (binding-site) heterogeneity.
Alternatively, if there is a concern about adjacent normal tissue exposure, then a short-path length $\alpha$-emitter may be more appropriate.

Radiation is well established as a known cell-killing modality. Keeping things simple is a hallmark of success on multiple fronts. The presence of cell surface targets and quantifiable exposure remove much of the mystery regarding the potential patient response. In addition, the ability to deliver cytotoxic payloads via extracellular binding obviates the need to address the multiple challenges associated with intracellular drug delivery (17). These radiopharmaceuticals are simple, stable constructs, and although targeted, the "target" is not the mechanism for cell death; it is only the conduit.

Repeated use of common isotopes in different radioligand therapies builds familiarity among clinicians and regulators, potentially accelerating both clinical development and commercial uptake.

\section{CONVINCING WALL STREET}

Momentum is building in the nuclear medicine field because of recent, high-profile developments in the radioligand therapy area. Advanced Accelerator Applications (AAA) paved the way for this new flood of innovation with the development of Lutathera (USAN: ${ }^{177} \mathrm{Lu}$-DOTATATE; INN: ${ }^{177} \mathrm{Lu}-\mathrm{OXODOTREOTIDE}$ ) (Advanced Accelerator Applications).

Funded largely by revenues from its radiopharmaceutical diagnostics business and investment from private family offices in Europe, AAA developed a robust global supply chain and manufacturing capability for ${ }^{177} \mathrm{Lu}$-DOTATATE, After successfully negotiating with regulators to leverage historical compassionate-use data in support of the conduct of a multicenter, international registration trial, the company raised more capital with existing investors and started positioning itself for listing on a major stock exchange to bolster anticipated investments in regulatory support and infrastructure building for commercial launch. Operations were established in the United States, and efforts to attract biotechnology equity investors were initiated. Some modest investments were secured; however, more than a decade of strong growth and financial performance in the radiopharmaceutical industry and pending data in a phase 3 registration study were not enough to overcome uncertainty regarding the future commercial success of radioligand therapy. AAA's bid for an initial public offering on the NASDAQ stock exchange was withdrawn in 2014 because of unfavorable pricing.

It was not until presentation of the compelling results of the phase 3 NETTER-1 trial during the presidential session at the September 2015 annual meeting of the European Society of Medical Oncologists that Wall Street gained confidence in the potential of radioligand therapy (18). After the disclosure of a $79 \%$ reduction in the risk of disease progression or death for patients who had somatostatin receptor-positive gastroenteropancreatic neuroendocrine tumors and who were treated with ${ }^{177} \mathrm{Lu}$-DOTATATE and supportive care (30 $\mathrm{mg}$ of octreotide [long-acting release]), compared with the results obtained with $60 \mathrm{mg}$ of octreotide (long-acting release) alone (Fig. 1) (18), AAA raised more than $\$ 75$ million in an oversubscribed initial public offering with highly sought-after biotechnology funds (19). Investors again showed their support in October 2016, when AAA raised more than $\$ 150$ million in a follow-on offering (20). (The most common and most serious adverse reactions observed in the NETTER-1 


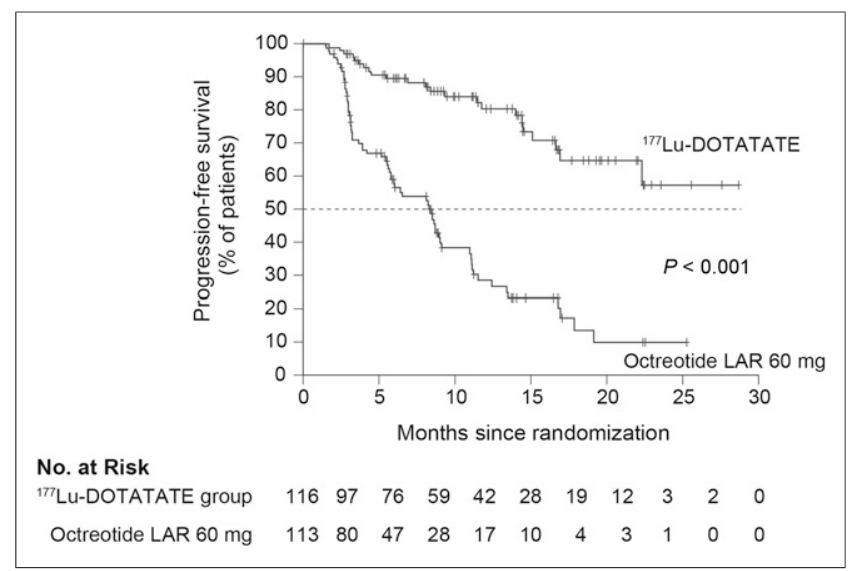

FIGURE 1. Kaplan-Meier curves for progression-free survival in NETTER-1 trial. LAR = long-acting release. (Adapted with permission of (18).)

trial were lymphopenia, increased gamma-glutamyltransferase level, vomiting, nausea, elevated aspartate aminotransferase level, increased alanine aminotransferase level, hyperglycemia, and hypokalemia (18)).

After some delays in the regulatory process, AAA achieved marketing authorization for ${ }^{177} \mathrm{Lu}$-DOTATATE in Europe in September 2017 and U.S. Food and Drug Administration approval in early 2018. In parallel, AAA was acquired by Novartis in a transaction valued at $\$ 3.9$ billion, more than double the company's valuation less than $1 \mathrm{y}$ before, validating the potential of radioligand therapy in the eyes of Wall Street (21). Further confirmation would come from initial reported revenues after the launch of ${ }^{177} \mathrm{Lu}$-DOTATATE in the United States - evidencing rapid uptake, successful reimbursement, and broad distribution among treating institutions-despite the complexities of a multidisciplinary

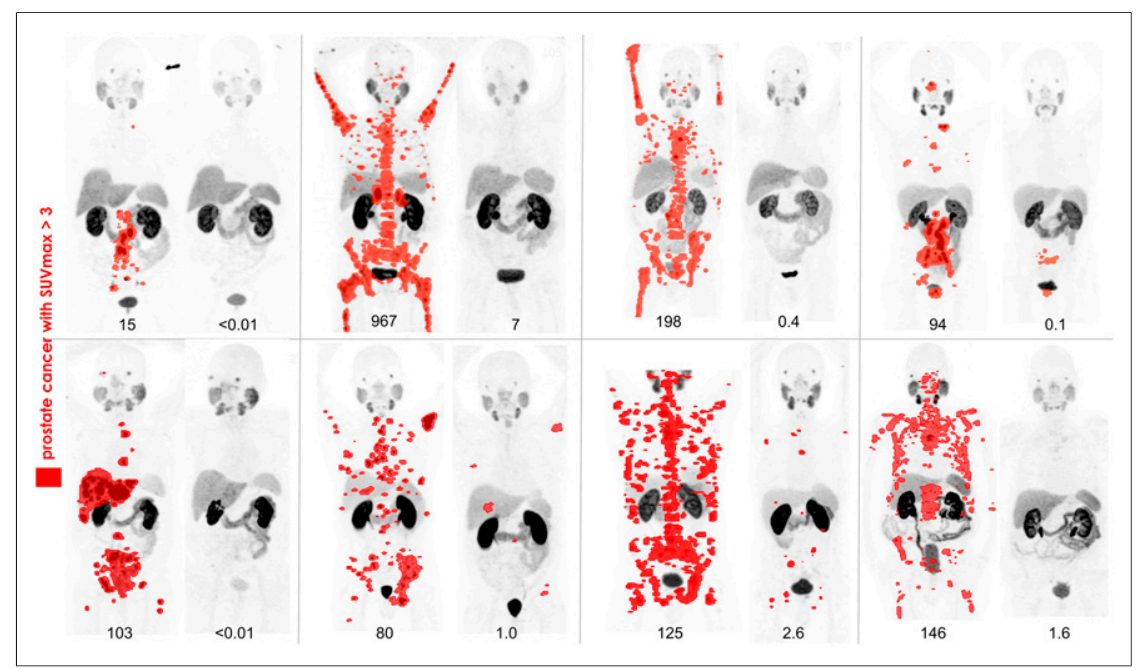

FIGURE 2. SNMMI Image of the Year: PSMA PET Imaging of Theranostic for Advanced Prostate Cancer. Shown are PSMA PET images before and after ${ }^{177}$ Lu-PSMA- 617 theranostic in 8 patients who had metastatic prostate cancer and for whom standard therapeutic options had been exhausted. ${ }^{68} \mathrm{Ga}-\mathrm{PSMA}-11$ PET maximum-intensity-projection images were obtained at baseline and 3 mo after ${ }^{177}$ Lu-PSMA-617 theranostic in 8 patients with prostate-specific antigen decline of $\geq 98 \%$ (values below images, in $\mathrm{ng} / \mathrm{mL}$ ) in prospective phase 2 study. Any disease with SUV $\max$ of $>3$ is shown in red. approach and an orphan indication. In early 2019, the American Society of Clinical Oncology recognized the treatment of rare cancers as the "Advance of the Year" and cited ${ }^{177}$ Lu-DOTATATE as an example (22).

In the autumn of 2017, Endocyte acquired the rights to ${ }^{177} \mathrm{Lu}-$ PSMA-617, another radioligand therapy with a much broader targeting indication-prostate cancer (23). Investors were initially skeptical, recognizing several significant potential hurdles previously navigated by AAA. It was not obvious to investors that this model could be repeated, in part because of AAA's significant previous experience in nuclear medicine.

Over the course of the next $12 \mathrm{mo}$, however, investors witnessed rapid momentum in the Endocyte ${ }^{177}$ Lu-PSMA-617 program. Endocyte established contract manufacturing capabilities, developed partnerships with oncology and nuclear medicine experts, and successfully solicited regulatory support for a registration trial in prostate cancer. Similar to AAA's path, Endocyte's path was accelerated by the ability to cite previously established clinical data. Endocyte had the benefit of compelling phase 2 data from a single-arm investigator-initiated study. Theranostic images generated during a phase 2 trial of ${ }^{177}$ Lu-PSMA-617 in patients with prostate-specific membrane antigen (PSMA)positive metastatic prostate cancer, conducted by Dr. Michael Hofman at the Peter MacCallum Cancer Centre, Melbourne, Victoria, Australia, garnered recognition as "Image of the Year" at the 2018 Society of Nuclear Medicine and Molecular Imaging conference (Fig. 2) (24,25). Endocyte's regulatory engagement also likely benefited from the approval of ${ }^{177} \mathrm{Lu}$-DOTATATE earlier in the same year.

Site engagement for the phase 3 VISION study of ${ }^{177} \mathrm{Lu}-$ PSMA-617 was rapid and enthusiastic, supported by the interdisciplinary approach already established in the treatment of prostate cancer. Bayer's efforts to educate the market about ${ }^{223}$ Ra-dichloride (Xofigo; Bayer) was likely a contributing factor. Investors soon became encouraged and started to recognize the potential for a repeat of the AAA model, allowing Endocyte to raise more than $\$ 270$ million to support development between March and September 2018 $(26,27)$. Less than one year after the closing of its acquisition of AAA, Novartis acquired Endocyte for a $\$ 2.1$ billion valuation, rewarding investors (28). It is worth noting that although Novartis made a significant investment in establishing its leadership position in radioligand therapeutics and nuclear medicine, other large pharmaceutical companies are also taking an increased interest in this therapeutic class, as evidenced by their participation in the Endocyte sale process.

\section{KEEPING THE OPPORTUNITY ALIVE}

As more data on promising targets and ligands in new indications continue to emerge, investors and pharmaceutical companies will be looking for the next radioligand success story. This excitement is being amplified by studies of potential combinations of radioligand therapies 
with other mechanisms of action, such as immunotherapies (29) or poly adenosine diphosphate ribose polymerase inhibitors that interfere with DNA repair (30).

With this emerging opportunity comes new responsibility for the nuclear medicine sector. Embedding radioligand therapies in oncology practice and the hearts and minds of investors will require increased discipline across the field, including focus on the following key development areas.

\section{Focusing on Innovation That Can Improve Therapeutic Outcomes}

Emphasis on single-target theranostic approaches that feature diagnostic imaging alongside new treatment options, such as radioligand therapy, should be increased. Investors (such as payers) attach greater value to technologies that have a measurable impact on health care outcomes.

\section{Increasing Discipline and Industry Participation in Early Clinical Development}

Once targets are validated, radioligand therapies have the potential to offer less development risk than other oncology approaches. However, this strategy requires more robust initial trial design and data gathering regarding comparative safety and efficacy.

\section{Strengthening of Collaboration Between Nuclear Medicine and Oncology}

The best development paths will be defined through interdisciplinary collaboration and enhanced knowledge of therapeutic options for a particular malignancy. Such partnerships may also facilitate institutional investment and payer support and may optimize patient care.

\section{Accelerating Radionuclide Supply Chain Development}

The full potential of this therapeutic approach will be realized only when the supply chain for more radionuclides is established. A lower-cost, high-quality reliable supply (i.e., a supply chain with geographic diversity and redundancy) will be critical for long-term success in larger indications. The establishment of such a supply chain will require public and private collaboration.

\section{Regulatory and Reimbursement Support}

Raising the profile of the impact of these therapies on patient outcomes will continue to be important in promoting a regulatory and reimbursement environment that supports continued investment and innovation. One critical aspect of this task is partnering with government regulators and payers to address the practice of local compounding at institutions once a commercially approved and manufactured radioligand therapy is available on the market. As industry participation (backed by investment) and support during early development increase, the need for local compounding should diminish over time.

\section{CONCLUSION}

Wall Street and large pharmaceutical companies are now aware of the potential of recent innovations in the nuclear medicine field, and investment is expected to continue. If the potential of radioligand therapy is to be truly realized and if the role of nuclear medicine in the treatment of cancer is to be elevated, then deliberation and discipline in the pursuit of the next promising targets are needed. There is an urgency to this work because patients are waiting.

\section{DISCLOSURE}

Mike Sherman is engaged as an advisor to Endocyte, Inc., and Rachel Levine is an employee of Advanced Accelerator Applications. No other potential conflict of interest relevant to this article was reported.

\section{REFERENCES}

1. Pusey WA. Roentgen-rays in the treatment of skin diseases and for the removal of hair. J Cutan Dis Syph. 1900;18:302-318.

2. Lederman M. The early history of radiotherapy 1895-1939. Int J Radiat Oncol Biol Phys. 1981;7:639-648.

3. Evolution of cancer treatments: radiation. American Cancer Society website. https:// www.cancer.org/cancer/cancer-basics/history-of-cancer/cancer-treatmentradiation.html. Updated June 12, 2014. Accessed May 8, 2019.

4. Silberstein EB. Radioiodine: the classic theranostic agent. Semin Nucl Med. 2012;42:164-170.

5. Levine R, Krenning EP. Clinical history of the theranostic radionuclide approach to neuroendocrine tumors and other types of cancer: historical review based on an interview of Eric P. Krenning by Rachel Levine. J Nucl Med. 2017;58(suppl): 3S-9S.

6. Ter-Pogossian MM, Phelps ME, Hoffman EJ, Mullani NA. A positron-emission transaxial tomograph for nuclear imaging (PET). Radiology. 1975;114: 89-98.

7. Ibritumomab tiuxetan. National Cancer Institute website. https://www.cancer. gov/about-cancer/treatment/drugs/ibritumomabtiuxetan. Updated July 25, 2018. Accessed May 8, 2019.

8. Bexxar [package insert] Research Triangle Park, NC: GlaxoSmithKline; 2012

9. Major phase 3 trial shows Zevalin extends progression-free survival in one of the most common types of lymphoma [press release]. FierceBiotech website. https://www.fiercebiotech.com/biotech/press-release-major-phase-3-trial-showszevalin-extends-progression-free-survival-one-of. Updated November 12, 2007. Accessed May 8, 2019.

10. Meredith RF, Knox S. Clinical development of radioimmunotheray for B-cell nonHodgkin's lymphoma. Int J Radiat Oncol Biol Phys. 2006;66(suppl):S15-S22.

11. Reno J. An effective treatment for a common cancer in the US may vanish from the market. Yahoo Finance. https://finance.yahoo.com/news/non-hodgkinlymphoma-zevalin-120931920.html. Published June 16, 2016. Accessed May 8, 2019.

12. Timmerman L. Why good drugs sometimes fail: the Bexxar story. Xconomy. https:/xconomy.com/national/2013/08/26/why-good-drugs-sometimes-fail-inthe-market-the-bexxar-story/. Published August 26, 2013. Accessed May 8, 2019.

13. Müller I. Guidelines for the successful generation of protein-ligand complex crystals. Acta Crystallogr D Struct Biol. 2017;73:79-92.

14. Marvin JS, Hellinga HW. Manipulation of ligand binding affinity by exploitation of conformational coupling. Nat Struct Biol. 2001;8:795-798.

15. Dash A, Pillai MRA, Knapp FF. Production of ${ }^{177} \mathrm{Lu}$ for targeted radionuclide therapy: available options. Nucl Med Mol Imaging. 2015;49:85-107.

16. Waaijer SJH, Kok IC, Eisses B, et al. Molecular imaging in cancer drug development. J Nucl Med. 2018;59:726-732.

17. Stewart MP, Lorenz A, Dahlman J, Sahay G. Challenges in carrier-mediated intracellular delivery: moving beyond endosomal barriers. Wiley Interdiscip Rev Nanomed Nanobiotechnol. 2016;8:465-478.

18. Strosberg J, El-Haddad G, Wolin E, et al. Phase 3 trial of ${ }^{177} \mathrm{Lu}$-Dotatate for midgut neuroendocrine tumors. N Engl J Med. 2017;376:125-135.

19. Lawrence S. Advanced Accelerator Applications up $25 \%$ on $\$ 75 \mathrm{M}$ IPO, as SurgiQuest files for IPO. FierceBiotech website. https://www.fiercebiotech. com/medical-devices/advanced-accelerator-applications-up-25-75m-ipo-assurgiquest-files-for-ipo. Published November 11, 2015. Accessed May 8, 2019.

20. Advanced Accelerator Applications S.A. announces pricing of $\$ 150$ million public offering of American depositary shares [press release]. Advanced Accelerator Applications website. https://www.adacap.com/advanced-accelerator-applications-sa-announces-pricing-of-150-million-public-offering-of-american-depositary-shares/. Accessed May 8, 2019

21. Novartis announces the planned acquisition of Advanced Accelerator Applications to strengthen oncology portfolio [press release]. Novartis website. https:// www.novartis.com/news/media-releases/novartis-announces-planned-acquisitionadvanced-accelerator-applications. Published October 30, 2017. Accessed May 8, 2019. 
22. Advance of the Year. American Society of Clinical Oncology website. https:// www.asco.org/research-progress/reports-studies/clinical-cancer-advances-2019/ advance-year. Accessed May 8, 2019.

23. Endocyte announces exclusive worldwide license of phase 3 ready PSMA-targeted radioligand therapy for development in prostate cancer [press release]. Endocyte, Inc., website. https://investor.endocyte.com/news-releases/news-releasedetails/endocyte-announces-exclusive-worldwide-license-phase-3-ready. Published October 2, 2017. Accessed May 8, 2019.

24. SNMMI Image of the Year: PSMA PET Imaging of Theranostic for Advanced Prostate Cancer. Society of Nuclear Medicine and Molecular Imaging website. http://www.snmmi.org/NewsPublications/NewsDetail.aspx? ItemNumber=29483. Updated June 26, 2018. Accessed May 8, 2019.

25. Hofman M, Violet J, Sandhu S, et al. High activity, pain reduction and low toxicity with Lutetium-177 PSMA617 theranostics in metastatic castrate-resistant prostate cancer (mCRPC): results of a phase II prospective trial [abstract]. J Nucl Med. 2018;59(suppl 1):531.

26. Endocyte announces closing of public offering of common stock [press release]. Endocyte, Inc., website. https://investor.endocyte.com/news-releases/news-release- details/endocyte-announces-closing-public-offering-common-stock. Published March 2, 2018. Accessed May 8, 2019.

27. Endocyte announces closing of public offering of common stock [press release]. Endocyte, Inc., website. https://investor.endocyte.com/news-releases/news-releasedetails/endocyte-announces-closing-public-offering-common-stock-0. Published September 14, 2018. Accessed May 8, 2019.

28. Novartis announces planned acquisition of Endocyte to expand expertise in radiopharmaceuticals and build on commitment to transformational therapeutic platforms [press release]. Novartis website. https://www.novartis.com/news/media-releases/novartis-announces-planned-acquisition-endocyte-expand-expertiseradiopharmaceuticals-and-build-commitment-transformational-therapeutic-platforms. Published October 18, 2018. Accessed May 8, 2019.

29. Kim C, Subramaniam DS, Liu SV, Giaccone G. Phase I/II trial of anti-PD-1 checkpoint inhibitor nivolumab and ${ }^{177} \mathrm{Lu}-\mathrm{DOTA}{ }^{0}-\mathrm{Tyr}^{3}$-Octreotate for patients with extensive-stage small cell lung cancer [abstract]. J Clin Oncol. 2018;36(suppl):TPS8589.

30. Nonnekens J, van Kranenburg M, Beerens CE, et al. Potentiation of peptide receptor radionuclide therapy by the PARP inhibitor Olaparib. Theranostics. 2016;6:1821-1832. 